\title{
Size Selective Synthesis of Colloidal Platinum Nanoparticles for Use as High Resolution EM Labels
}

\author{
D.A. Meyer and R.M. Albrecht
}

Department of Animal Sciences, University of Wisconsin, Madison, WI 53706

In the 30 years since Faulk and Taylor introduced the immunogold technique [1], labeling with colloidal gold (cAu) particles has become the predominant method for electron microscopy (EM). Frens subsequently developed a procedure to prepare monodisperse Au suspensions over a broad range of particle diameters [2], which enabled multiple labeling with cAu particles of different sizes. However, the number of different cAu labels that can be used simultaneously for multiple labeling is limited by a number of factors. The minimum particle diameter is determined by the instrument resolution, and each label must correspond to a set of particles with average diameters sufficiently distinct from one another to prevent size overlap and confusion between different labels. As the diameter increases, larger particles decrease the spatial resolution of the labeling and sterically hinder other particles from gaining access to their attachment sites. Both of these factors decrease the labeling efficiency. In order to address these concerns, recent efforts in this lab have led to the development of two novel approaches to EM multiple labeling, employing colloidal particles of similar sizes but of different shapes and elemental compositions [3].

The drawbacks inherent with using colloidal metal particles of different sizes for multiple labeling do not negate the utility of having available particles in a wide range of sizes in order to maximize the versatility of the labeling technology. Frens demonstrated with cAu that exquisite control over the average particle diameter could be achieved by means of a standard condensation technique in which gold chloride is reduced by varying concentrations of trisodium citrate in aqueous solution. The citrate reduces Au atoms to zero valency, which then nucleate. Subsequent growth of the colloidal particles occurs as the remainder of the Au atoms in solution crystallizes around the nuclei. Higher citrate concentrations maximize the rate of nucleation relative to crystallization, leading to the formation of a large number of nuclei and, hence, particles with smaller diameters. On the other hand, lower concentrations of the reducing agent induce the formation of smaller numbers of gold nuclei and particles with larger diameters. However, Frens' citrate method has thus far proven inadequate for the synthesis of colloidal particles composed of metals other than Au over a broad range of particle diameters. Like the citrate method, other techniques that control the independent processes of nucleation and crystallization, such as manipulation of the reaction temperature, work only for specific metals, in this case palladium.

One promising, simple, and practical avenue for the size-controlled synthesis of colloidal particles, composed of a potentially wide variety of different metals, was developed by Zsigmondy during the early years of the past century [4]. His method involves the reduction of gold chloride in the presence of previously synthesized cAu particles one to three nanometers in size that act as nuclei around which additional Au crystallizes. Particle size is controlled by varying the concentration of cAu nuclei relative to the Au salt concentration, much as Frens controlled particle size by adjusting the amount of reducing agent added. Voigt and Heumann applied Zsigmondy's method to the formation of uniform $\mathrm{cAg}$ particles [5]. We have recently utilized this approach to prepare $\mathrm{cPt}$ particles of varying diameters by reducing aqueous solutions of chloroplatinic acid with tannic acid in the presence of cPt nuclear particles with an average diameter of $2.5 \mathrm{~nm}$ (table 1 and figure 1). Future work will apply this method to other noble metals, including $\mathrm{Pd}, \mathrm{Rh}$, and $\mathrm{Ru}$. 
Of course, Zsigmondy's nuclear method is not limited solely to the synthesis of colloidal particles composed of single metals. In order to develop nanoparticles with enhanced catalytic properties, researchers in this field have prepared bimetallic clusters using similar techniques as well as coreduction processes [6,7]. Such bimetallic particles might also have application in the field of EM labeling if they prove to be distinguishable from their homogenous counterparts by analytic means, such as electron energy loss spectroscopy, as we have shown previously for particles composed of single metals [3].

\section{References}

[1] W.P. Faulk and G.M. Taylor, Immunochemistry 8 (1971) 1081.

[2] G. Frens, Nature Physical Science 241 (1973) 20.

[3] D.A. Meyer and R.M. Albrecht, Microscopy and Microanalysis 7 (Suppl. 2) (2001) 1032.

[4] R.A. Zsigmondy, Zeitschrift für Anorganische Chemie 99 (1917) 105.

[5] J. Voigt and J. Heumann, Zeitschrift für Anorganische Chemie 169 (1928) 140.

[6] A. Henglein, Journal of Physical Chemistry B 104 (2000) 6683.

[7] J.H. Hodak et al., Journal of Chemical Physics 114 (2001) 2760.

TABLE 1. Average diameters of cPt particles prepared with varying amounts of $\mathrm{cPt}$ nuclear sol. For sols $1-5,100 \mathrm{ml} \mathrm{cPt}$ was prepared by adding nuclear sol to solutions of $500 \mu \mathrm{M} \mathrm{H}_{2} \mathrm{PtCl}_{6}$ stabilized with $0.04 \%$ trisodium citrate and reduced with $0.05 \%$ tannic acid at $100^{\circ} \mathrm{C}$.

\begin{tabular}{c|c|c}
\hline Sol & Volume Nuclear Sol Added $(\mathbf{m l})$ & Average Particle Diameter $(\mathbf{n m})$ \\
\hline Nuc. Sol & - & $2.5 \pm 0.03$ \\
1 & 10.0 & $5.5 \pm 0.06$ \\
2 & 5.0 & $7.1 \pm 0.06$ \\
3 & 2.5 & $9.0 \pm 0.09$ \\
4 & 1.0 & $11.6 \pm 0.12$ \\
5 & 0.5 & $13.4 \pm 0.16$ \\
\hline
\end{tabular}
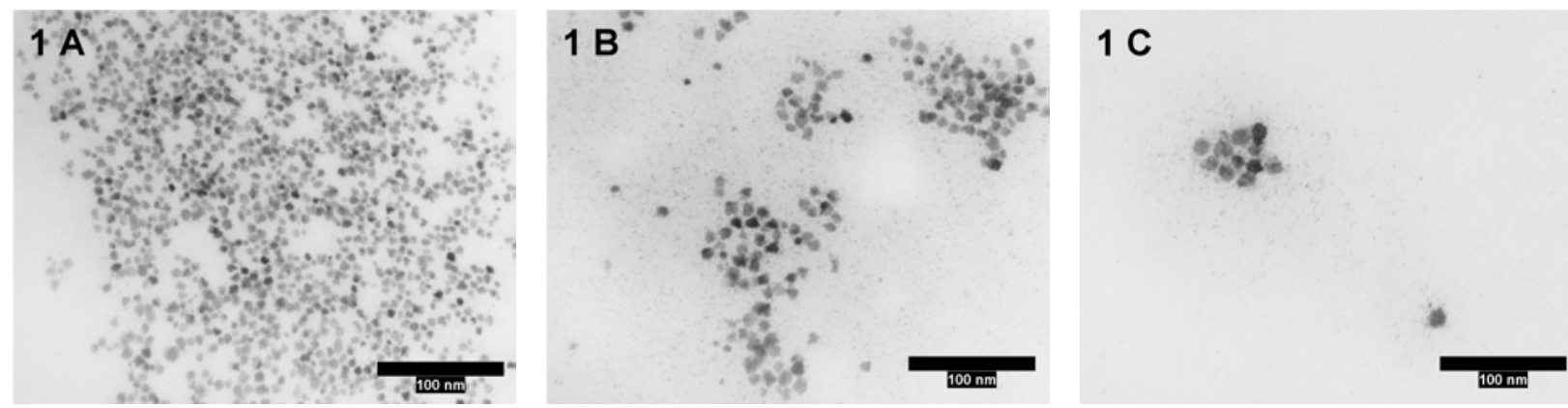

Figure 1. Transmission electron micrographs showing cPt particles prepared by reduction of $500 \mu \mathrm{M}$ $\mathrm{H}_{2} \mathrm{PtCl}_{6}$ at $100^{\circ} \mathrm{C}$ with $0.05 \%$ tannic acid and stabilized with $0.04 \%$ trisodium citrate. Various volumes of nuclear sol were added prior to reduction, and the total reaction volume in each case was $100 \mathrm{ml}$. Magnification in each image is 230,000 times at an accelerating voltage of $80 \mathrm{KeV}$. Bars are each $100 \mathrm{~nm}$. 1A. cPt particles prepared in the presence of $10 \mathrm{ml}$ nuclear sol. Average particle diameter is $5.5 \mathrm{~nm}$. 1B. cPt particles prepared in the presence of $2.5 \mathrm{ml}$ nuclear sol. Average particle diameter is $9.0 \mathrm{~nm}$. 1C. cPt particles prepared in the presence of $0.5 \mathrm{ml}$ nuclear sol. Average particle diameter is $13.4 \mathrm{~nm}$. 